\title{
Parâmetros psicométricos de testes psicológicos de inteligência
}

\author{
Ana Paula Porto Noronha \\ Fernanda Andrade Freitas \\ Fernanda Ottati \\ Universidade São Francisco
}

\begin{abstract}
Resumo
O presente estudo abordou a construção dos testes psicológicos, em especial no que se refere à padronização e aos estudos de validade e precisão. O objetivo da pesquisa foi verificar a presença ou a ausência de validade e precisão nos testes de inteligência, bem como identificar se os testes estrangeiros possuem padronização brasileira. Foram analisados 26 testes de inteligência comercializados no Brasil, e os resultados indicaram que grande parte dos testes nacionais e dos internacionais apresenta estudos de validação e de precisão, embora nem todos tenham indicado nos respectivos manuais, os dados referentes à padronização, como amostra de padronização e variáveis relacionadas. Sugere-se a realização de outros estudos com diferentes testes ou novas análises sobre os testes de inteligência.
\end{abstract}

Palavras chave: avaliação psicológica; precisão e validade; construção de instrumentos.

\begin{abstract}
Psychometrics parameters of tests of intellingence

The aim of the present study was to analyze the making of psychological tests, focusing on standardization and studies of validity and reliability. The objective of the research was to verify whether the tests of intelligence are valid and reliable or not, as well as to identify if the foreign tests present Brazilian standardization. Twenty six of the national tests of intelligence commercialized in Brazil were analyzed and the results indicated that most tests, national and international ones, present studies of validation and reliability, although some of them have not mentioned information concerning standardization, in the respective manual, as a sample of standardization and related variables. New studies are recommended, focusing on different tests or new analysis of the tests of intelligence.
\end{abstract}

Keywords: psychological assessment; reliability and validity; construction of instruments.

Construir testes psicológicos não é uma atividade simples, pois a construção é um processo que demanda tempo, que requer domínios específicos, como por exemplo, Psicometria e Estatística e que consiste na execução de um plano de atividades, que compreende uma série de etapas. Para Adánez (1999) as etapas da construção de um teste são as seguintes: definição dos objetivos do teste, especificação do contexto, eleição do modelo matemático, definição do domínio, construção dos itens e das instruções, revisão da primeira versão por expertos, estudo piloto, seleção das amostras e aplicação do teste inicial, análise e seleção empírica dos itens, avaliação da precisão e da validade do teste, elaboração de normas e redação final do manual em uso.

No seu trabalho, o autor faz uma descrição de cada uma das etapas consideradas importantes quando da construção e aponta que os manuais considerados clássicos de Psicometria não tratam o tema de forma equilibrada, ou seja, atribuem mais importância a um determinado conteúdo, em detrimento de outros, sendo que estudos que supram essa carência teórica devem ser publicados para minimizar as dificuldades. Em contrapartida, na realidade nacional, a situação é ainda mais grave, considerando que são poucos os manuais, clássicos, ou não, que se destinam à discussão da construção dos testes psicológicos.

Para Oakland (1999) os padrões que determinam a construção dos testes devem ser estabelecidos, de maneira que possam ser utilizados em qualquer parte do mundo. $\mathrm{O}$ autor define oito passos para o desenvolvimento dos testes, a saber: identificar a necessidade e definir as qualidades que serão testadas, obter um contrato para o teste, iniciar a elaboração dos itens, iniciar a coleta dos dados do pré-teste e a respectiva análise, realizar a coleta para a padronização do teste e a respectiva análise e preparar o teste para a distribuição. 
O autor discute detalhadamente cada um dos passos elencados e afirma que a apresentação desse trabalho tem como objetivo, além de discutir a construção dos testes psicológicos, de forma a propiciar o desenvolvimento deles, encorajar os interessados a ingressarem na atividade. Em comum, os trabalhos apontam para o rigor que a construção de um instrumento requer e alertam que todas as etapas ou passos devem ser atendidos. A questão que se coloca diante da construção dos instrumentos disponíveis no mercado profissional brasileiro é se os instrumentos construídos atenderam às especificações tratadas nos estudos abordados.

A American Educational Research Association AERA, a American Psychological Association - APA e o National Council on Measurement in Education NCME (1999) publicaram um guia sobre os padrões para os testes psicológicos e educacionais. O objetivo da obra é providenciar critérios para a avaliação e a prática dos testes e para verificar os efeitos do uso. Embora a AERA, a APA e o NMCE destaquem que o standards deve servir apenas como uma referência e que outros materiais devem ser desenvolvidos, não se encontram com frequiência materiais como esse. No capítulo que se destina à construção, avaliação e documentação, há uma extensa discussão sobre as qualidades psicométricas dos instrumentos (validade e precisão).

Outro aspecto merecedor de destaque é a revisão dos instrumentos. Salvia e Ysseldyke (1991) apresentam orientações que objetivam facilitar a análise. $\mathrm{O}$ trabalho, apesar de modesto, contribui com a área à medida que procura sistematizar uma prática, que, no caso do Brasil, ainda conta com poucas sistematizações, como a avaliação psicológica. Os autores abordam questões como a validade e a precisão, os escores do teste, ou ainda, a clareza das informações contidas nos manuais. E oferecem sugestões práticas para a revisão, do tipo "descobrir os dados sobre confiabilidade pode ser mais difícil. Se houver uma seção sobre a confiabilidade, a tarefa será extremamente simples. Você deseja ver se há evidências de cada tipo de confiabilidade adequada. Exija números; não aceite declarações sobre a confiabilidade do teste" (p. 590).

O tema é relevante, pois instrumentos são usados na sua versão original, sem que tenham se submetido a estudos freqüentes de revisão e atualização. Recentemente, o Conselho Federal de Psicologia (CFP, 2001) publicou a Resolução $25 / 2001$, na qual apresenta diretrizes para a elaboração, a comercialização e o uso dos testes psicológicos. Ela determina que sejam atingidos os seguintes critérios mínimos para a elaboração de instrumentos: apresentação da fundamentação teórica do instrumento, enfatizando a definição do construto, e descrevendo-o em seus aspectos constitutivo e operacional; apresentação da validade e da pre- cisão, justificando os procedimentos específicos adotados na investigação; apresentação de dados sobre as propriedades psicométricas dos itens do instrumento; apresentação do sistema de correção e interpretação dos resultados. Mais especificamente em relação à revisão dos instrumentos, a resolução aponta: "os dados empíricos das propriedades de um teste psicológico devem ser revisados periodicamente, não podendo a revisão ser realizada após período de 10 (dez) anos, isto é, o intervalo entre um estudo e outro com o objetivo de revisar os dados dos instrumentos não pode ser superior a 10 anos" (s. p.).

Outros autores vêm apontando a importância da revisão dos instrumentos. Figueiredo e Pinheiro (1998) apontam que os critérios utilizados na validação do instrumento podem mudar através do tempo e que os testes além de serem válidos e precisos, para a garantia do valor científico, devem ser padronizados para um grupo sociocultural específico.

A revisão de um teste é uma tarefa fundamental para que o instrumento continue oferecendo a confiabilidade necessária a qualquer instrumento psicológico. As revisões devem ser realizadas periodicamente, embora pouco se saiba ainda sobre o "prazo de validade" de cada teste, considerando que isso varia de acordo com o construto medido, com o tipo de teste, com o material e com tantos outros aspectos que, apesar de merecedores de destaque, não serão abordados nesse trabalho. A AERA, APA e NMCE (1999) indicam que as revisões são necessárias quando novos dados de pesquisa, mudanças significativas no domínio ou novas condições para o uso e interpretação revelam que o teste não é mais apropriado para seu uso.

A Comissão Internacional de Testes e o Colégio Oficial de Psicólogos (ITC, 2001) estabeleceram diretrizes internacionais para o uso dos testes. O trabalho versa sobre o uso, sobre a construção e sobre a revisão. No que se refere à construção, o documento aponta que os psicólogos que constróem os instrumentos utilizam procedimentos científicos e conhecimentos profissionais adequados para a determinação dos métodos, padronização, validação e precisão.

\section{Parâmetros Psicométricos}

Os instrumentos psicológicos são instrumentos de medida e, por isso, devem apresentar características que justifiquem sua confiabilidade. Nos testes psicológicos são duas as características verificadas para que se ateste a legitimidade e a confiabilidade deles (Pasquali, 2001): validade e precisão.

Costuma-se dizer que um teste é válido quando ele mede o que supostamente deve medir, e, segundo a AERA, APA e NMCE (1999), a validade é a conside- 
ração mais fundamental quando da construção e análise dos testes, pois revela o quanto a evidência e o suporte teórico do teste estão absorvidos na proposta apresentada.

A fidedignidade de um teste refere-se ao quanto o resultado obtido pelo sujeito se aproxima do resultado verdadeiro do sujeito num traço qualquer (Pasquali, 2001). Ela tem sido analisada à luz da quantidade de erros que está presente nos resultados do teste, de forma que quando eles apresentam uma pequena quantidade de erros, considera-se a medida confiável (Adánez, 1999). Obter os coeficientes de precisão de um referido instrumento é necessário e primordial para se atestar o grau de confiabilidade da medida.

A AERA, APA e NMCE (1999) apontam que a fidedignidade refere-se à consistência dos dados de avaliação frente aos mesmos procedimentos de testagem em indivíduos ou em grupos, quando as condições de padronização são mantidas. A informação sobre o erro de medida é indispensável para a própria avaliação e para o uso do instrumento.

A precisão e a validade são conceitos importantes, pois de alguma forma atestam a qualidade do instrumento. Os métodos disponíveis para a obtenção da validade e da precisão são variados e cada instrumento deve escolher com cautela os métodos mais adequados às suas características. Em relação à precisão, as técnicas de demonstração mais utilizadas são testereteste, formas paralelas e metades. E no que se refere à validade, as três grandes técnicas clássicas são: validade de construto, validade de conteúdo e validade de critério. Na realidade brasileira tem havido uma preocupação em viabilizar o trabalho do psicólogo, não somente através da informatização e do aprimoramento de tecnologias (Costa, 2000), como também através da cautela quanto aos estudos de precisão e de validade.

O presente trabalho objetiva analisar, dentre testes de inteligência, a presença de estudos sobre validade e precisão, além da ocorrência de padronização brasileira, no caso de testes estrangeiros.

\section{MÉTODO}

\section{Instrumentos Consultados}

Para a realização do presente estudo foram consultados 26 testes psicológicos que avaliam inteligência, a saber:

1. BPR-5 Bateria de Provas de Raciocínio (R. Primi \& L. S. Almeida, Casa do Psicólogo, 1998)

2. Bateria TSP (C. Piovani, Edites, sem data)
3. Cubos de Kohs (O. Rainho \& C. C. Ribeiro, CEPA, sem data)

4. D-48 Dominós (E. Nick, CEPA, 1966)

5. WISC Escala de Inteligência Wechsler para Crianças (A. M. Poppovic, CEPA, 1964)

6. Escala de Maturidade Mental Columbia (I. C. B. Alves, J. L. Duarte \& W. Duarte, Casa do Psicólogo, 1993)

7. G-36 Teste de Inteligência Não-Verbal (N. E. R. Boccalandro, Vetor, 1966)

8. G-38 Teste de Inteligência Não-Verbal (N. E. R. Boccalandro, Vetor, 1979)

9. Medida de Fluência Verbal (C. Piovani, Edites, sem data)

10. Desenho da Figura Humana (S. M. Wechsler, 1998)

11. INV (P. Weil \& E. Nick, CEPA, 1951)

12. Prova de Nível Mental (J. Maya, CEPA, 1949)

13. Teste Barcelona (Técnicos do ISOP da Fundação Getúlio Vargas, CEPA, sem data)

14. CIA Teste Coletivo de Inteligência para Adultos (R. Moraes, E. M. Andrade \& D. G.Alves, CEPA, sem data)

15. Teste de Capacidades Intelectuais (C. Del Nero, Vetor, sem data)

16. HTM Teste de Habilidade para o Trabalho Mental (L. M. C. Santarosa, O. Wainstein \& Z. R. Prado, Vetor, 1983)

17. Teste D-70 ( G. C. Amaral, Casa do Psicólogo, 1988)

18. Teste de Sondagem Intelectual (J. A. Cunha, M. I. B. Moraes, N. S. Rocha, L. Werba, R. R. Valle, M. Spadir, J. Moraes \& I. Salermo, CEPA, sem data)

19. Teste Eqüicultural de Inteligência (E. M. Andrade \& D. G. Alves, CEPA, sem data)

20. Teste dos Relógios (F. V. Souza \& S. V. Cambrais, Vetor, 1967)

21. Teste Edites de Inteligência - T.E.I. (C. M. Piovani \& C. Piovani, Edites, sem data)

22. R-1 Teste Não-Verbal de Inteligência (R. Oliveira, Vetor, sem data)

23. V-47 Teste Verbal de Inteligência (N. E. R. Boccalandro, Vetor, 1971)

24. Matrizes Progressivas escala geral (F. Campos, CEPA, 1997)

25. Matrizes Progressivas escala avançada (F. Campos, CEPA, sem data) 
26. Matrizes Progressivas Coloridas (A. Angelini, I. C. B. Alves, E. M. Custódio \& W. M. Duarte, Casa do Psicólogo, 1988).

Vale destacar que as referências encontradas entre os parênteses referem-se aos dados de identificação do material analisado. Alguns dos testes não possuíam datas em seus manuais, o que inviabilizou a análise de acordo com a época de construção deles.

\section{CRITÉRIOS DE AVALIAÇÃO}

Esse tipo de trabalho permite muitos tipos diferentes de análise, no entanto, o presente estudo procurou verificar a presença ou ausência de estudos relacionados à validade e precisão quando da construção dos testes e da elaboração dos manuais. Além disso, verificou se havia padronização, sendo que, para os testes estrangeiros, verificou-se a existência de padronização brasileira. Os testes foram analisados individualmente, e os dados foram organizados em uma planilha para posterior análise.

\section{RESULTADOS E DISCUSSÃO}

Inicialmente é necessário esclarecer que os 26 testes analisados não esgotam a totalidade no que se refere à avaliação da inteligência, embora atenda à grande parte dos instrumentos disponíveis do mercado nacional. Os resultados estão apresentados na Tabela 1; a primeira coluna refere-se à identificação dos testes, a segunda, a terceira e a quarta coluna referem-se aos dados de validade, precisão e padronização respectivamente. Os critérios usados nessas análises foram presença (1 ponto) ou ausência (0) dos elementos analisados. A última coluna traz a data do manual consultado e a procedência do material (nacional ou estrangeiro).

Dos 26 testes, $61,54 \%(\mathrm{~N}=16)$ são publicações nacionais, ou seja, instrumentos construídos no Brasil, enquanto $38,46 \%(\mathrm{~N}=10)$ referem-se a instrumentos estrangeiros que foram traduzidos, adaptados e/ou padronizados para a realidade brasileira. Esse dado revela um aspecto bastante positivo no desenvolvimento da ciência psicológica, relacionado ao necessário crescimento de produção nacional e ao aumento dos estudos com amostras regionais que tratam as peculiaridades das diferentes populações do Brasil. Não são poucos os autores que indicaram a urgência na criação de materiais originalmente nacionais (Almeida, 1999; Wechsler, 1999; Van Kolch, 1981).

Ainda em relação aos instrumentos nacionais e estrangeiros, dos instrumentos nacionais quase metade não especificou no manual a data de sua publicação $(43,75 \%, F=7)$, o que fere orientações internacionais quanto aos cuidados que se deve ter quando da elaboração dos manuais de testes (Oakland, 1999; ITC, 2001; Adánez, 1999). Nos instrumentos estrangeiros, a porcentagem aproximou-se da anterior $(40 \%, \mathrm{~F}=4)$. 
Tabela 1 - Validade, precisão e padronização de testes de inteligência

\begin{tabular}{|c|c|c|c|c|}
\hline Nome dos testes & Validade & Precisão & Padronização & Produção \\
\hline Prova de Nível Mental & 1 & 0 & 1 & $\mathrm{~N}(1949)$ \\
\hline INV & 1 & 1 & 1 & N (1951) \\
\hline G-36 Teste de Inteligência Não-Verbal & 1 & 1 & 1 & N (1966) \\
\hline Teste dos Relógios & 0 & 0 & 0 & N (1967) \\
\hline V-47 Teste Verbal de Inteligência & 1 & 1 & 0 & N (1971) \\
\hline G-38 Teste de Inteligência Não-Verbal & 1 & 1 & 1 & N (1979) \\
\hline HTM Teste de Habilidade para o Trabalho Mental & 1 & 1 & 1 & $N(1983)$ \\
\hline Desenho da Figura Humana & 1 & 1 & 1 & N (1998) \\
\hline BPR-5 Bateria de Provas de Raciocínio & 1 & 1 & 1 & N (1998) \\
\hline R-1 Teste Não-Verbal de Inteligência & 1 & 1 & 1 & $N(s$. d. $)$ \\
\hline Teste Edites de Inteligência - T.E.I. & 0 & 1 & 0 & $N($ s. d.) \\
\hline Bateria TSP & 1 & 1 & 1 & $N(s . d)$. \\
\hline Teste Barcelona & 0 & 0 & 1 & $N(s$. d. $)$ \\
\hline Teste de Sondagem Intelectual & 1 & 1 & 1 & $N(s$. d. $)$ \\
\hline Teste de Capacidades Intelectuais & 0 & 0 & 0 & $N(s . d)$. \\
\hline Medida de Fluência Verbal & 1 & 1 & 1 & $N(s . d)$. \\
\hline WISC Escala de Inteligência Wechsler para Crianças & 1 & 1 & 0 & $E(1964)$ \\
\hline D-48 Dominós & 1 & 1 & 0 & $E(1966)$ \\
\hline Teste D-70 & 0 & 1 & 1 & $E(1985)$ \\
\hline Matrizes Progressivas Coloridas & 1 & 1 & 1 & $E(1988)$ \\
\hline Escala de Maturidade Mental Columbia & 1 & 1 & 1 & E (1993) \\
\hline Matrizes Progressivas escala geral & 1 & 1 & 1 & E (1997) \\
\hline Teste Eqüicultural de Inteligência & 1 & 1 & 1 & $E$ (s. d.) \\
\hline Cubos de Kohs & 1 & 0 & 0 & $E$ (s. d.) \\
\hline Matrizes Progressivas escala avançada & 1 & 1 & 0 & $E(s . d)$. \\
\hline CIA Teste Coletivo de Inteligência para Adultos & 1 & 1 & 1 & $E(s . d)$. \\
\hline
\end{tabular}

$\mathrm{Ne} \mathrm{E}$ representam respectivamente, testes nacionais e estrangeiros. As datas que seguem a letra são referentes à data de publicação do manual avaliado. A abreviatura s.d. está sendo usada nos casos em que o manual não apresenta data de publicação.

No que se refere à década de publicação, observouse nos instrumentos nacionais uma distribuição a partir da década de 40 até 90 , o que não foi observado nos instrumentos estrangeiros; eles aparecem na década de 60 e se fortalecem a partir de 80 . No entanto, para que essa análise não se torne parcial, não se pode desconsiderar que o presente estudo se faz num contexto sócio-histórico em que instrumentos do início da Psicometria no Brasil já não se encontram mais disponíveis para aquisição e não são encontrados em todos os centros universitários, como os citados no trabalho de Pasquali e Alchieri (2001), que objetivou fazer uma contextualização histórica dos testes psicológicos. Os autores apresentaram uma relação dos instrumentos produzidos a partir de 1930 no Brasil, tais como ABC (M. B. Lourenço Filho, 1931), PMK (E. Mira y Lopez, 1940), Teste Jota (O. A. L. Martins, 1944), Minhas Mãos (H. Antipoff, 1947), Classificação de Objetos do ISOP (E. Schneider, 1950), Dadahie (E. M. Andrade, R. Moraes \& Z. S. Wend,
1950), Coordenação Manual (D. Antipoff, 1951) e DEP (O. B. Barros, 1952).

Em relação à padronização dos instrumentos, dos 16 testes construídos no Brasil, 4 (25\%) não informam, nos respectivos manuais, a amostra utilizada, os estudos realizados, as variáveis escolhidas para a análise, e outros dados relacionados à padronização. Nos instrumentos estrangeiros, a porcentagem é $40 \%$ $(\mathrm{F}=4)$. A informação sobre os cuidados tomados na padronização do teste é imprescindível para que os usuários possam escolher os instrumentos de acordo com as características para as quais eles foram construídos (Adánez, 1999).

No que se refere à validade, $12(75 \%)$ dos testes nacionais têm esse tipo de estudo, enquanto que nos testes estrangeiros, 9 (90\%) desenvolveram algum estudo. Dos testes nacionais, $12(75 \%)$ testes apresentam estudos de precisão e, nos estrangeiros, 9 (90\%). Embora os valores apresentados na validade e na precisão dos testes nacionais e estrangeiros tenham sido os mesmos, vale ressaltar que os testes variaram, 
ou seja, alguns possuem estudos de precisão e não de validade.

$\mathrm{Na}$ análise geral dos testes, é possível encontrar aqueles, cujos manuais não apresentam informações sobre a padronização, sobre a validade e sobre a precisão, como por exemplo, Teste dos Relógios e Teste de Capacidades Intelectuais, contrariando todas as orientações recebidas pelos manuais de Psicometria (Van Kolch, 1981; Anastasi \& Urbina, 2001).

Finalizando, os instrumentos foram organizados de acordo com as respectivas datas de construção e, apesar de muitos instrumentos não portarem a data de publicação, foi possível observar na amostra estudada que os instrumentos nacionais a partir da década de 70 apresentaram padronização e os parâmetros psicométricos. Nos estrangeiros, a mesma observação se fez presente, no entanto, a partir da década de 80 .

\section{CONSIDERAÇÕES FINAIS}

Algumas das questões que poderiam ser discutidas nessa seção já foram abordadas nos resultados. Reserva-se às considerações finais a ênfase de que é imprescindível que um maior rigor permeie a construção, a padronização e a revisão dos testes psicológicos no Brasil. É urgente que a Resolução 25/2001 do Conselho Federal de Psicologia (CFP, 2001), que apresenta diretrizes para a elaboração, comercialização e para o uso dos testes psicológicos seja colocada em prática. Ela determina que sejam atingidos determinados critérios para a elaboração de instrumentos, dentre eles a apresentação da validade e da precisão, justificando os procedimentos específicos adotado na investigação, e cuidados em relação à padronização.

Embora os números desse estudo não tenham trazido surpresa e não sejam mais assustadores, de alguma forma foi possível constatar que ainda existem instrumentos, utilizados na prática profissional do psicólogo, que não apresentam garantias de validade e de precisão, ou seja, que não garantem a confiabilidade dos resultados que oferecem. Essa situação chama a atenção, porque segundo a AERA, APA e NMCE (1999), a validade é a consideração mais fundamental quando da construção e análise dos testes, pois revela o quanto à evidência e o suporte teórico do teste estão absorvidos na proposta apresentada, e a informação sobre o erro de medida é indispensável para a própria avaliação e para o uso do instrumento. Não promover estudos nesse sentido é não garantir se o instrumento atendeu às expectativas iniciais.

No que se refere à produção de instrumentos, o estudo revelou que a produção nacional já vem ganhando espaço e que, dentre a amostra analisada, se apresenta em número superior ao número de testes estrangeiros. Também observou-se que houve um tímido, mas crescente aumento de número de instrumentos a partir das décadas de 70/80.

Pesquisas sobre os instrumentos de medida devem continuar, de forma a propiciar melhores instrumentos, com mais qualidade, que ofereçam dados confiáveis e que garantam bons processos de avaliação psicológica. Esse estudo modestamente revelou a existência ou a ausência de dados de padronização, validade e precisão nos manuais de testes. Novos estudos devem abordar outros instrumentos, ou ainda novos estudos devem ser desenvolvidos com os testes de inteligência.

\section{REFERÊNCIAS}

Adánez, G. P. (1999). Procedimientos de construcción y análisis de tests psicométricos. Em S. M. Wechsler \& R. S. L. Guzzo (Orgs.), Avaliação psicológica: Perspectiva internacional (p. 57-100). São Paulo: Casa do Psicólogo.

Almeida, L. S. (1999). Avaliação psicológica: Exigências e desenvolvimentos nos seus métodos. Em S. M. Wechsler \& R. S. L. Guzzo (Orgs.), Avaliação psicológica: Perspectiva internacional (p. 41-55). São Paulo: Casa do Psicólogo.

American Educational Research Association, American Association \& National Council on Measurement in Education (1999). Standards for educational and psychological testing. New York: American Educational Research Association.

Anastasi, A. \& Urbina, S. (2000). Testagem psicológica. Porto Alegre: Artes Médicas.

Conselho Federal de Psicologia - CFP (2001). Resolução 25/2001. http://www.pol.org.br. Obtido em: 28 dez. 2001

Costa, F. R. (2000). Informatização de testes psicopedagógicos: Metodologias, uso e discussões éticas. Anais do V Encontro Mineiro de Avaliação Psicológica: Teorização e prática, VIII Conferência Internacional Avaliação Psicológica: Formas e contextos (p. 103), Belo Horizonte: PUC-MG.

Figueiredo, V. L. M. \& Pinheiro, S. (1998). O Teste WISCIII em uma amostra do Rio Grande do Sul. Temas em Psicologia, 6(3), 255-261.

ITC - Comisión Internacional de Tests (2001). Diretrices internacionales para el uso de los tests. http://www. cop.es/tests/Diretrices.htm>. Obtido em: 04 dez. 2001.

Oakland, T. (1999). Developing standardized tests. Em S. M. Wechsler \& R. S. L. Guzzo (Orgs.), Avaliação psicológica: Perspectiva internacional (p. 101-118). São Paulo: Casa do Psicólogo.

Pasquali, L. (2001). Parâmetros psicométricos dos testes psicológicos. Em L. Pasquali (Org.), Técnicas do exame psicológico: TEP Manual, Volume I: Fundamentos das técnicas psicológicas (p. 111-136). São Paulo: Casa do Psicólogo.

Pasquali, L. \& Alchieri, J. C. (2001). Os testes psicológicos no Brasil. Em L. Pasquali (Org.), Técnicas do exame psicológico: TEP Manual, Volume I: Fundamentos das técnicas psicológicas (p. 195-221). São Paulo: Casa do Psicólogo.

Salvia, J. \& Ysseldyke, J. (1991). Avaliação em educação especial e corretiva. São Paulo: Manole Editora. 
Van Kolch, O L. (1981). Técnicas do exame psicológico e suas implicações no Brasil. Petrópolis: Vozes.

Wechsler, S. M. (1999). Guia de procedimentos éticos para a avaliação psicológica. Em S. M. Wechsler \& R. S. L. Guzzo (Orgs.), Avaliação psicológica: perspectiva internacional (p. 133-141). São Paulo: Casa do Psicólogo.

Recebido: 23.05 .2002

Revisado: 13.10 .2002 Aceito: 20.10 .2002

\section{Sobre as autoras}

Ana Paula Porto Noronha: Doutora em Psicologia: ciência e profissão, pela Pontifícia Universidade Católica de Campinas; docente da graduação e do Programa de Estudos Pós-graduados em Psicologia da Universidade São Francisco.

E-mail: ananoronha@saofrancisco.edu.br.

Fernanda Andrade Freitas: Bolsista de Iniciação Científica - PROBAIC/USF.

Fernanda Ottati: Auxiliar voluntária de pesquisa. 